\title{
Marine sponge-derived polymeric alkylpyridinium salts as a novel tumor chemotherapeutic targeting the cholinergic system in lung tumors
}

\author{
LAURA PALEARI ${ }^{1 *}$, SONYA TROMBINO ${ }^{2 *}$, CARLA FALUGI $^{2}$, LORENZO GALLUS $^{2}$, \\ SEBASTIANO CARLONE ${ }^{1}$, CRISTIANO ANGELINI ${ }^{2}$, KRISTINA SEPCIC $^{3}$, TOM TURK $^{3}$, \\ MARCO FAIMALI ${ }^{4}$, DOUGLAS M. NOONAN ${ }^{5}$ and ADRIANA ALBINI ${ }^{1}$ \\ ${ }^{1}$ Laboratory of Molecular Oncology, Department of Translational Oncology, National Institute for Cancer \\ Research (IST); ${ }^{2}$ Laboratory of Developmental Biology, Department of Biology, University of Genoa, Genoa; \\ ${ }^{3}$ Biotechnical Faculty, Department of Biology, University of Ljubljana, Ljubljana, Slovenia; ${ }^{4}$ Marine \\ Technology Section, Institute of Marine Sciences (ISMAR), National Research Council (CNR), Genoa; \\ ${ }^{5}$ Department of Clinical and Biological Sciences, University of Insubria, Varese, Italy
}

Received January 18, 2006; Accepted March 23, 2006

\begin{abstract}
Previous studies have shown that the cholinergic system plays a pivotal rule in small cell lung cancer (SCLC) cell growth through an autocrine loop that activates the nicotinic cholinergic receptor, which together with the activation of this receptor by nicotine links SCLC evolution with tobacco use. Non-small cell lung cancer (NSCLC) is the most common form of lung cancer and is also linked to tobacco use. Here we describe the presence of molecules of the cholinergic system in NSCLC samples and cell lines and investigate the implications of the cholinergic system in cell growth regulation. Cholinoacetyltransferase (ChAT), vesicular acetylcholine transporter (VAChT) and acetylcholinesterase (AChE) were observed in NSCLC tumor biopsies and in NSCLC cell lines. Polymeric alkylpyridinium salts (poly-APS) are AChE inhibitors isolated from the crude extract of the marine sponge, Reniera sarai. These metabolites were characterized as a mixture of two polymers of 3-octylpyridinium, including 29 and 99 monomeric
\end{abstract}

Correspondence to: Dr A. Albini, Laboratory of Molecular Oncology, Department of Translational Oncology, National Institute for Cancer Research (IST), Largo Rosanna Benzi 10, 16132 Genova, Italy

\section{${ }^{*}$ Contributed equally}

Abbreviations: ACh, acetylcholine; AChE, acetyl-cholinesterase (E.C. 3.1.1.7.); VAChT, vesicular acetylcholine transporter; ChAT, cholino-acetyltransferase; SCLC, small cell lung cancer; NSCLC, non-small cell lung cancer; poly-APS, polymeric alkylpyridinium salts

Key words: marine sponge, alkylpyridinium salts, cholinergic system, lung tumors units. Exposure of normal lung fibroblast and NSCLC cell lines to poly-APS revealed a selective cytotoxicity for cancer cells as compared to the normal fibroblast cell lines. FACS analysis indicated poly-APS induced apoptosis in NSCLC cells but not in normal lymphocytes. Non-toxic doses of poly-APS also potently reduced NSCLC cell-cell adhesion in suspension cultures. The limited toxicity of poly-APS on normal cells was confirmed by injection in the caudal vein of mice. No overt effects on health parameters, such as weight gain and physical behavior, were observed, and histological analysis of major organs did not reveal differences between the treated animals as compared to controls. These data demonstrate that NSCLC cells express cholinergic molecules that may be involved in cell growth regulation and that the cholinesterase inhibitor, poly-APS, shows selective toxicity toward NSCLC cells while having no apparent toxicity towards normal cells and tissue in vitro and in vivo.

\section{Introduction}

Lung cancer remains the leading cause of mortality due to cancer in the Western world. Of the two major types of lung cancer, approximately $75-80 \%$ are non-small cell lung cancer (NSCLC) with the remaining $20-25 \%$ being small cell lung cancer (SCLC). SCLC, a rapidly disseminating and metastatic cancer (1), is almost invariably linked to tobacco use (2). The effects of tobacco exposure are linked to the presence of nicotinic receptors, part of the cholinergic system, on SCLC cells (3). Small cell lung carcinomas express receptors for acetylcholine (ACh), and their stimulation by nicotine or other cholinergic agonists stimulates cell growth through activation of nicotinic cholinergic receptors (nAChRs) and/or muscarinic cholinergic receptors (mAChRs) (4-6). Cholinergic autocrine loops may also regulate SCLC cell growth (7), as these cells express cholino-acetyltransferase (ChAT), the vesicular ACh transporter (VAChT) and cholinergic receptors. 
Non-small cell lung cancer (NSCLC) is currently the most common cause of cancer mortality and morbidity extending beyond industrialized countries (8). Although NSCLC occurs in non-smokers, tobacco smoking is clearly a key etiological factor responsible for the majority of these cancers (8). The correlation between tobacco use and NSCLC incidence has been thoroughly demonstrated (9-11) but there is no direct evidence that the growth of this tumor is regulated by nicotine activation of cholinergic receptors.

The observation that $\mathrm{ACh}$ receptors (AChRs) can regulate lung cancer cell growth suggests that the cholinergic system may be a potential pharmaceutical target. Efficient inhibitors of acetylcholinesterase (AChE) have been isolated from the Adriatic marine sponge, Reniera sarai (12). These compounds were identified as polymeric 3-octylpyridinium salts (polyAPS) that exist as a mixture of two polymers with molecular weights of 5600 and 18900 Da. Poly-APS form part of a defense mechanism of the sponge against biofouling by other marine organisms. In laboratory conditions, they show an effective non-toxic anti-settlement effect on barnacles of Balanus amphitrite (13). In general, pyridinium derivates are known as AChE inhibitors acting either as competitive inhibitors binding on the catalytic anionic site at the bottom of the enzyme gorge, or as non-competitive inhibitors binding at the peripheral anionic site at the rime of the enzyme gorge, slowing or preventing the entrance of acetylcholine into the gorge $(14,15)$. In contrast, inhibition of AChE by a highly polymeric poly-APS proceeds by a rather complex and unusual pattern (16), ending in an irreversible inhibition of the enzyme (17). In addition to AChE-inhibition, poly-APS also exert haemolytic, and moderate and variable cytotoxic activity on different cell lines (16).

These observations led us to investigate the expression of the cholinergic system in NSCLC cells, and to then test the potential of poly-APS as a chemotherapy agent in lung cancer models. Here we show that NSCLC cell lines and cells isolated from primary NSCLC all express cholino-acetyltransferase (ChAT), the vesicular ACh transporter (VAChT) and acetylcholinesterase (AChE). Further, we show that the AChE inhibitors, poly-APS, have selective toxicity on tumor cells with log lower toxicity towards normal lung fibroblasts. These effects, combined with the fact that poly-APS are reasonably well-tolerated in vivo in animal models, indicate that these compounds may be considered as alternative inhibitors of lung cancer with lower systemic toxicity.

\section{Materials and methods}

Poly-APS. Poly-APS were isolated from Reniera sarai sponge according to previously described procedures (12). Stock concentrations of $1 \mathrm{mg} / \mathrm{ml}$ in distilled water were prepared. Based on activity, these stocks were stable at $4^{\circ} \mathrm{C}$ for at least several months. Since poly-APS compounds are a mixture of 2 polymers, the molar concentration calculations were estimated assuming a 1:1 ratio between them, giving a molecular weight of $12200 \mathrm{~g} / \mathrm{mol}$.

Surgical samples and cell lines. Selected surgical samples were obtained in an ethically compatible manner (information, anonymity, allowance etc., according to the European laws of Bioethics) from affected patients. NSCLC samples were cut for the classification according to the International Classification of Diseases for Oncology (ICD-O). Some samples were used for Western blot analysis as described below.

A549, Ca-Lu-1 and Sk-Lu-1 adenocarcinoma cell lines were grown in RPMI-1640 supplemented with $10 \%$ non-heat inactivated FBS, L-glutamine and appropriate antibiotics. MRC-5 normal lung fibroblast primary cell line was grown in DMEM supplemented with $10 \%$ FBS, L-glutamine, antibiotics and non-essential amino acids (Celbio).

Lymphocytes were obtained from healthy donor buffy-coats after Ficoll (Lympholyte-H, Cedarlane) separation and were cultured in RPMI-1640 supplemented with $10 \%$ non-heat inactivated FBS, L-glutamine and appropriate antibiotics.

\section{Methods}

Immunohistochemical analysis. Slides bearing A549 cultured cells were fixed with cold $4 \%$ paraformaldehyde (PFA) in phosphate-buffered saline (PBS), thoroughly rinsed and processed for immunohistochemical analysis against cholinergic molecules. Slides were incubated for $30 \mathrm{~min}$ at room temperature with the primary antibodies. Polyclonal anti-ChAT (Chemicon International) that was used to detect the biosynthetic enzyme for ACh. Anti-AChE polyclonal antibodies (Santa Cruz Biotechnology) were used to identify the lytic enzyme for ACh. Anti-VAChT polyclonal antibodies (Santa Cruz Biotechnology) were used to identify the vesicular transporter of ACh. Primary antibodies were diluted 1:250 in Tris- $\mathrm{HCl} 0.05 \mathrm{M}$ (pH 7.4) containing 1\% BSA. After incubation, samples were rinsed three times with Tris-buffered saline and $0.1 \%$ Tween-20 (TBST) and incubated for $30 \mathrm{~min}$ at room temperature with peroxidase-labeled polymer conjugated to goat anti-mouse or anti-rabbit immunoglobulins (EnVision+ System, Peroxidase; Dako Corporation). The provided substrate-chromogen was applied to cells and incubated for 5-10 min. Nuclei counterstaining was obtained with Mayer's hematoxylin (Bio-Optica) incubation. Stained slides were mounted in glycerol gel mounting media (Dako).

Western blot analysis. NSCLC samples, A549 and SK-Lu-1 cells were homogenized in buffer containing $0.1 \%$ Tween and $5 \mu \mathrm{l} / \mathrm{ml}$ of protease inhibitor cocktail (Sigma). The protein extracts were quantified with a Bradford-based colorimetric method (Bio-Rad). Total protein homogenate (20-30 $\mu \mathrm{g})$ was run into a $10 \%$ SDS-PAGE and then transferred to a nitrocellulose membrane. Non-specific binding sites were blocked with blocking buffer containing TBST with 5\% non-fat milk powder for $45 \mathrm{~min}$ at room temperature, and the blot was incubated with specific antibodies in blocking buffer with rabbit polyclonal anti-AChE (Santa Cruz Biotechnology), rabbit polyclonal anti-VAChT (Santa Cruz Biotechnology) rabbit polyclonal anti-ChAT (Chemicon International) at room temperature overnight. After washing, the blot was incubated with the appropriate secondary antibodies (Santa Cruz, Biotechnology) for $1 \mathrm{~h}$ and $30 \mathrm{~min}$ at room temperature. After extensive washing, detection was performed directly on the membrane using one-step NBT-BCIP solution (Pierce). All experiments were performed at least three times, obtaining very similar results. 
Cell proliferation assay. Poly-APS toxicity was tested using crystal violet staining. All of the experiments for each drug were performed at least three times with a minimum of 6 replicates per data point per experiment. Cells were plated with an 8-channel pipette at 1500 cells/well in 96-well plates. PolyAPS was added to plates $24 \mathrm{~h}$ after plating and experiments were carried out for 48,72 and 96 h respectively, in a range of concentration of $500 \mathrm{nM}$ to $1.28 \mathrm{pM}$.

Organ toxicity. To examine for organ toxicity, C57BL/6N mice ( $\mathrm{n}=30,20 \pm 2 \mathrm{~g}$ ) (Charles River Laboratories) were divided into five groups (6 per group) and injected with poly-APS through the caudal vein at $0.5 \mathrm{mg} / \mathrm{kg}$ and at $1 \mathrm{mg} / \mathrm{kg}$ with Heparin-sulphate (Heparin, Sigma-Italy, 10 U/100 g body weight). Animals were sacrificed at specific time intervals $(\mathrm{t}=0 ; 30 \mathrm{~min}, 1 \mathrm{~h}, 2 \mathrm{~h}, 24 \mathrm{~h})$ and the principal organs were taken immediately for routine histological analysis. The animals were treated according to the institute's animal care for the protection of vertebrates used for experimental and other specific purposes.

$3 D$ cultures. A549 cells $\left(2 \times 10^{6}\right)$ were plated in round-bottom bacterial 96-well plates and cultured in RPMI-1640 containing $20 \%$ of methyl-cellulose (stock $0.6 \mathrm{~g} / 50 \mathrm{ml}$ ) to induce growth suspension, with untreated cells forming spheroids. The cells were treated with $800 \mathrm{pM}$ of poly-APS for $24 \mathrm{~h}$ and analyzed morphologically with light and confocal microscopy.

Lectin staining. 3D A549 cultures were rinsed in PBS and placed in a humid chamber. To prevent internalization of the lectins the cells were treated with $0.02 \%$ sodium azide in PBS. Cells were then treated with wheat germ agglutinin (WGA) conjugated with fluorescein (F1TC) and concanavalin A (ConA) conjugated with rhodamine (TR1TC) diluted 1:10 for $30 \mathrm{~min}$ at room temperature, fixed with $4 \%$ formalin for $30 \mathrm{~min}$, and mounted using glycerol-saline for viewing with a confocal microscope.

Annexin-V staining. Following previously described protocols (18), the A549 adenocarcinoma cell line and normal (activated and non-activated) lymphocytes were treated for $24 \mathrm{~h}$ with $451 \mathrm{nM}$ of poly-APS and analyzed using a FACSort cytometer (Becton-Dickinson).

Statistical analysis. Data obtained from cytotoxicity test were statistically analyzed with Primer Program Version 1.0. The statistical analysis was performed by ANOVA followed by Student's-Newman-Keuls tests for multiple comparison and significance between differences was established at $\mathrm{P}<0.05$.

\section{Results}

Presence of cholinergic molecules in NSCLC. Previous studies have shown that molecules of the cholinergic system are expressed on and influence the growth of SCLC, and suggested a role for nicotine as a promoting agent in tobacco-driven lung cancer development. We therefore investigated whether this system is present and active in NSCLC. Western blot analyses demonstrated expression of the components of the cholinergic system on NSCLC tumor cell lines in vitro and

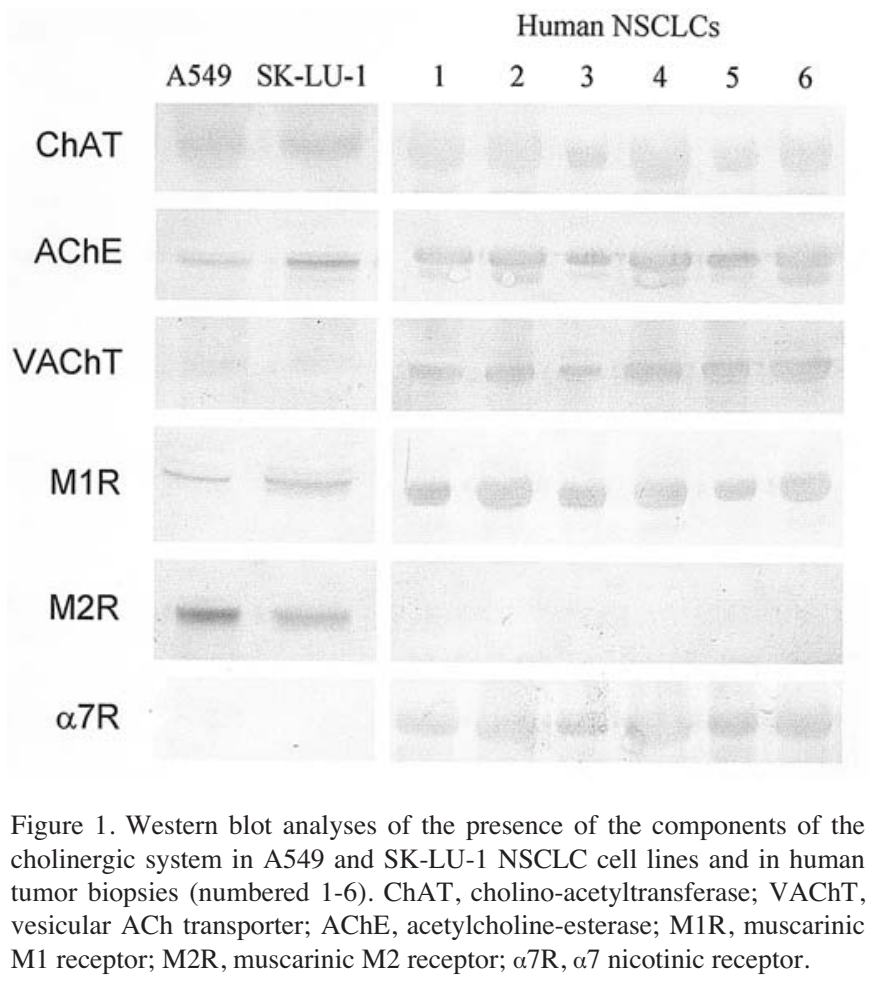

in NSCLC tumor biopsies in vivo (Fig. 1). Enzymes involved in production of the transmitter were strongly expressed in both cell ines and tumor biopsy samples, including cholinoacetyltransferase (ChAT), and vesicular ACh transporter (VAChT). Both cell lines and tumor biopsy samples showed expression of the lytic enzyme of ACh, acetylcholine-esterase (AChE) (Fig. 1). While both cell lines and tumor biopsies showed strong expression of the G-protein coupled muscarinic M1 class receptor, the cell lines showed expression of the M2 class muscarinic receptor, which regulates ion channels, whereas the tumor biopsies did not (Fig. 1). In contrast, only the tumor biopsies showed expression of the $\alpha 7$ nicotinic receptor, while the cultured cell lines were negative. The reason for these differences is not clear, but may reflect adaptation to culture conditions on one hand and a promotion effect by nicotine in lung cancer on the other.

In cultured A549 cells, the ChAT enzyme was mainly localized in the cytoplasm, showing an intense activity of synthesis of the enzyme, diffusing towards the periphery. The staining extended to the membrane only in some focal points of cell-to-cell contact (Fig. 2), in particular areas of cell-tocell adhesion were immunoreactive. The ACh transporters VAChT in A549 cells immuno-localized in vesicles that were mainly distributed on the cytoplasmatic side of the cell surface, suggesting that ACh is actively transported outside the cell (Fig. 2). Taken together, these data demonstrate autonomous synthesis and release of acetylcholine by NSCLC cells.

Immunocytochemistry with specific AChE polyclonal antibodies demonstrated prominent intracellular immunoreactivity in A549 cells. The anti-AChE staining pattern was consistent with vesicular localization; in some cases, the enzyme is localized in the cytosolic surface of the cell membrane (Fig. 2). Expression of the ACh lytic enzyme 


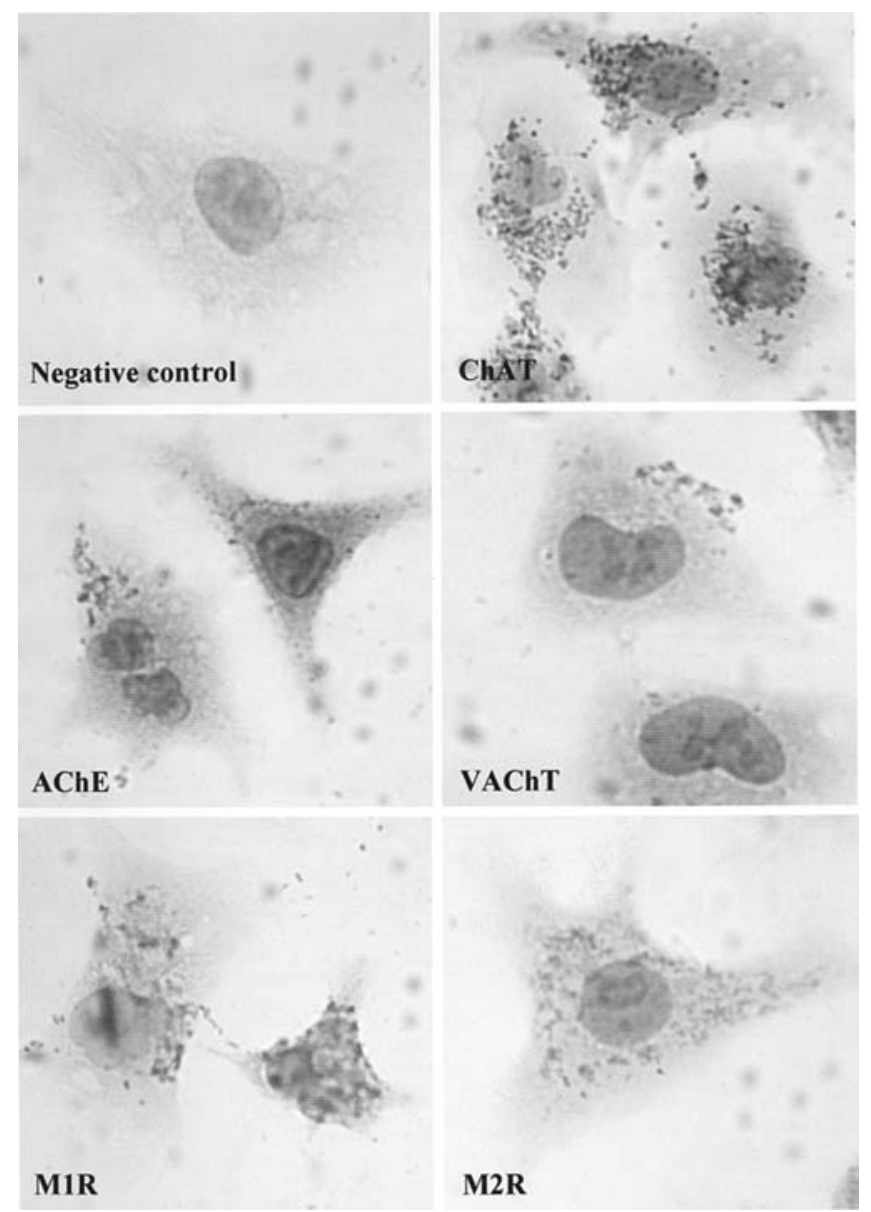

Figure 2. Immunohistochemical analysis to detect the localization of cholinergic components in A549 cells in vitro. ChAT, cholino-acetyltransferase; VAChT, vesicular ACh transporter; AChE, acetylcholineesterase; M1R, muscarinic M1 receptor; M2R, muscarinic M2 receptor.

indicates tight regulation of ACh activity in NSCLC. The class 1 muscarinic receptors were clearly localized on cultured A549 cells (Fig. 2), while reactivity for the class 2 muscarinic receptors was weaker and for the $\alpha 7$ nicotinic receptor it was absent (not shown).

Interruption of cholinergic regulation induces cytostasis/ cytotoxicity. Since molecules that pertain to the cholinergic system are expressed in both NSCLC lines and tumor tissues, we investigated the effects of altering cholinergic homeostasis in cell growth and viability in vitro. We used poly-APS as an $\mathrm{AChE}$ inhibitor to induce accumulation of $\mathrm{ACh}$ in vitro in normal lung fibroblasts, NSCLC cell lines and primary NSCLC cell cultures. The dose- and time-dependent responses to polyAPS were determined using the crystal violet assay. Poly-APS selectively inhibited tumor cell growth in a concentrationdependent manner (Fig. 3). While MRC5 lung fibroblasts did not show alterations in growth until high concentrations of poly-APS were used, at which point cell viability rapidly declined, showing an $\mathrm{EC}_{50} \mathrm{~s}$ of $50.27 \mathrm{nM}(72 \mathrm{~h})$ and $50.66 \mathrm{nM}$ (96 h) . In contrast, the A549 and CaLu NSCLC cell lines showed higher sensitivity to poly-APS, with $\mathrm{EC}_{50}$ s one $\log$ lower on the order of 4.41-6.9 nM. Finally, primary cultures of NSCLC cells were exquisitely sensitive to the cell killing

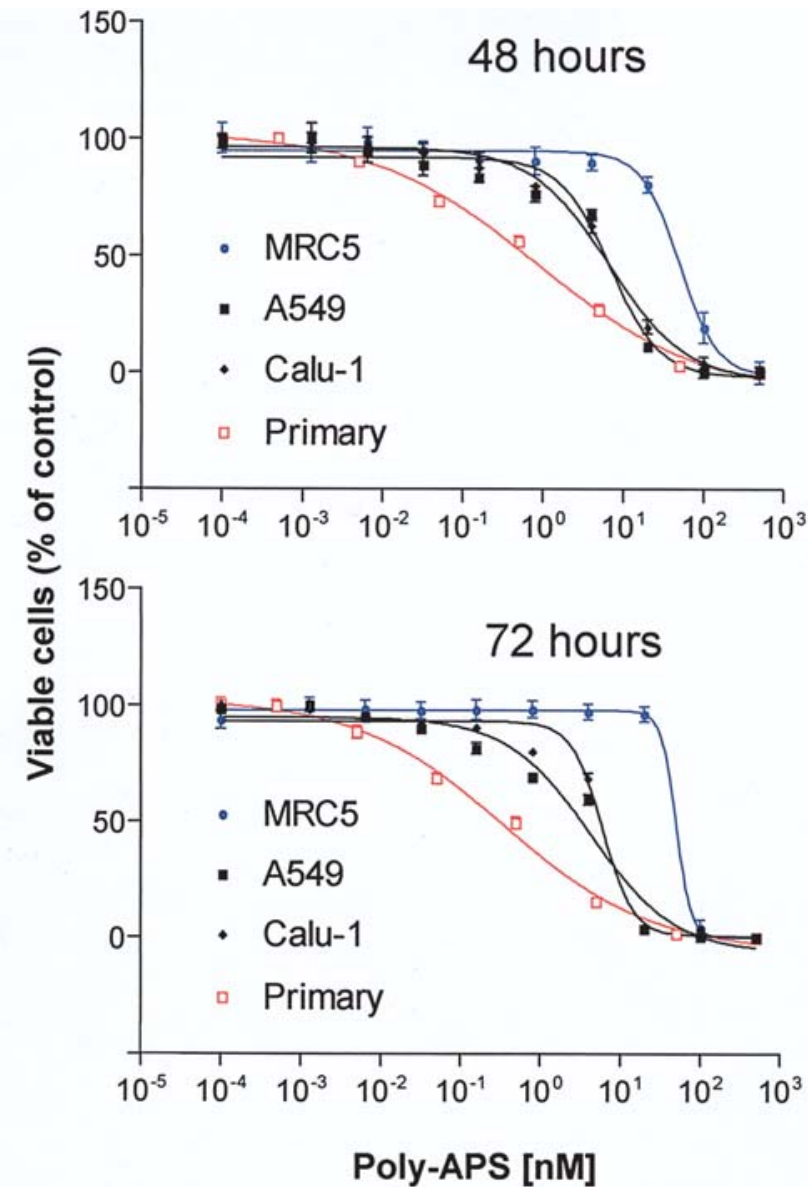

Figure 3. Selective dose-dependent inhibition of tumor cell proliferation in vitro by poly-APS. MRC5, the MRC5 human primary lung fibroblast line. A549, A549 NSCLC cells. Calu-1, Calu-1 NSCLC cells. Primary, a primary NSCLC cell culture. Tests were carried out for 48 and $72 \mathrm{~h}$ at the indicated concentrations. Bars indicate means \pm SEM.

activity of poly-APS, with $\mathrm{EC}_{50} \mathrm{~s}$ of 0.86 and $0.36 \mathrm{nM}$, fully two logs lower than that of normal lung fibroblasts. While the Hill slopes were relatively steep for MRC5 fibroblasts, suggesting a toxicity threshold, those for the primary cells were relatively shallow, indicating an alteration in biological signaling that leads to cell death by apoptosis. These data showed that poly-APS inhibition of AChE led to potent growth inhibition and cell death in NSCLC cells but had more limited effects on normal cells.

Quantification of apoptosis in normal and tumor cells. The activation of apoptosis by poly-APS in normal and tumor cells was quantitatively analyzed by FITC-labeled annexin V/ propidium iodide (PI) uptake analysis with fluorescent cytometry. In the early stages of apoptosis, membrane phosphatidylserine (PS) is translocated from the inner to the outer plasma membrane, exposing PS to the binding of annexin V (18). PI stains nucleic acids and does not enter into viable cells, thus PI-positive cells are indicative of an apoptotic or necrotic event. Vehicle-treated normal leukocytes or A549 tumor cells (controls) were primarily annexin V-FITC and PI-negative, indicating that the cells were viable and not apoptotic (Fig. 4). Freshly isolated normal leukocytes were used to verify the relatively low toxicity of poly-APS for normal cells. Even 


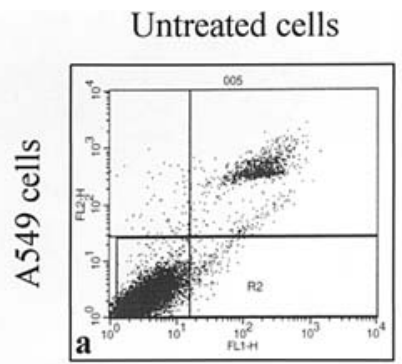

Poly-APS treated cells
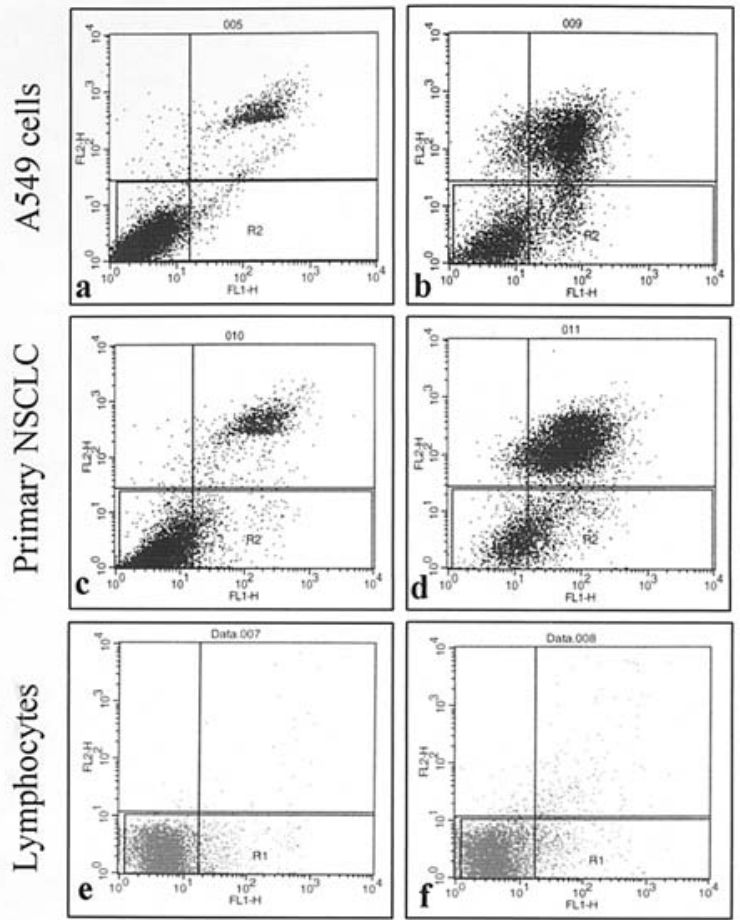

\begin{tabular}{|c|c|c|c|c|c|c|}
\hline g & 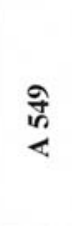 & 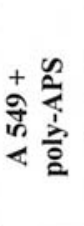 & 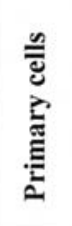 & 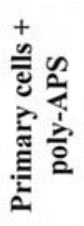 & 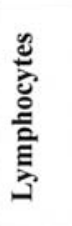 & 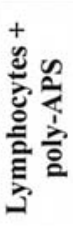 \\
\hline $\begin{array}{l}\text { PI+/Annexin V- } \\
\text { (Necrosis) }\end{array}$ & 0.64 & 5.10 & 0.42 & 3.18 & 0.16 & 1.09 \\
\hline $\begin{array}{c}\text { PI+/Annexin V+ } \\
\text { (late apoptosis) }\end{array}$ & 12.38 & 53.51 & 14.52 & 72.32 & 0.35 & 2.84 \\
\hline $\begin{array}{c}\text { PI-/Annexin V- } \\
\text { (Viable) }\end{array}$ & 85.13 & 31.77 & 79.09 & 13.72 & 93.42 & 89.14 \\
\hline $\begin{array}{l}\text { PI-/Annexin V+ } \\
\text { (early apoptosis) }\end{array}$ & 1.85 & 5.96 & 5.97 & 10.78 & 6.06 & 6.93 \\
\hline
\end{tabular}

Figure 4. Induction of apoptosis in A549 NSCLC lines, primary NSCLC cells and in normal lymphocytes (as indicated) by analysis of Annexin $\mathrm{V}$ by flow cytometry. Control (a, c and e) and poly-APS-treated cells (b, d and f). a and b, A549 cells; c and d, primary cells; e and f, freashly isolated CD ${ }^{+}$ lymphocytes. $\mathrm{g}$, The table indicates the percentage of gated cells in the single cell populations.

after treatment with relatively high $(450 \mathrm{nM})$ levels of polyAPS for $24 \mathrm{~h}$, normal leukocytes showed low levels of apoptosis only slightly above those of untreated cells (Fig. 4). Leukocytes activated with Phaseolus vulgaris Leucoagglutinin (PHA-H) showed only a slight increase in sensitivity to apoptosis (data not shown), gating on CD3 indicated that most of these few apoptotic cells were CD3-positive. In contrast, and in agreement with in vitro studies, a strong increase in annexin V binding was observed in A549 cells after exposure to poly-APS, with nearly $60 \%$ of the cells in apoptosis (Fig. 4). Again, primary NSCLC cells showed even higher sensitivity to poly-APS, with $\sim 80 \%$ of the cells in apoptosis. These data indicate that lung cancer cells are particularly sensitive to apoptosis induction upon interference with the cholinergic system by poly-APS, while control cells were not.

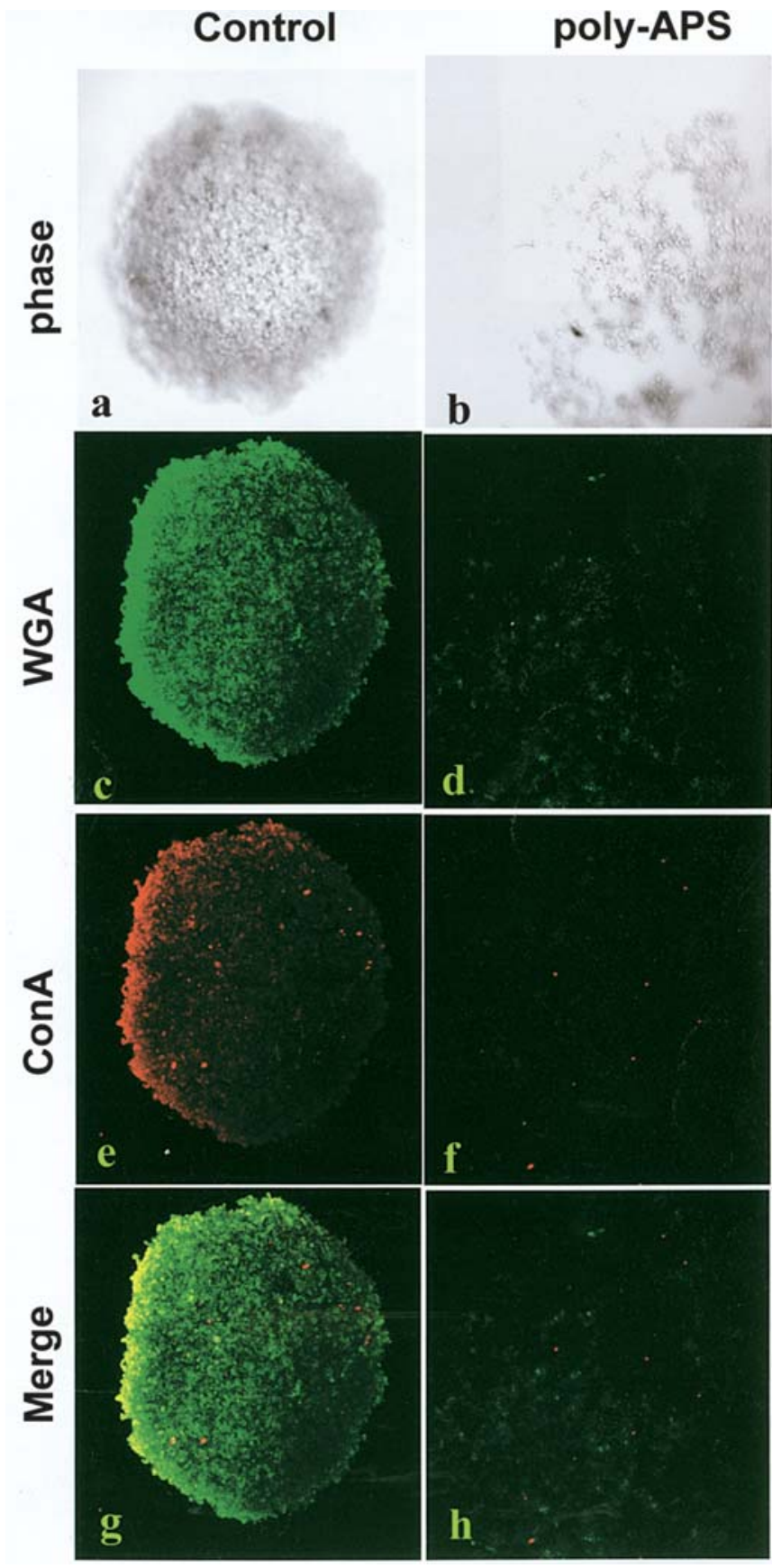

Figure 5. Three-dimensional spheroids formed by A549 cells either untreated or sub-lethal poly-APS treated. WGA and ConA lectin staining and merge of both on control and treated cells as indicated.

Effect of low poly-APS doses on cell-cell interatins in vitro. When grown in suspension culture, NSCLC cell lines formed three-dimensional spheroids with tight cell-cell adhesion. When these cultures were treated with low, sub-toxic doses of polyAPS, a remarkable change in morphology was observed, with inhibition of the ability of the cells to form spheroids, the cells remained scattered on the bottom of the wells (Fig. 5). Malignant transformation has been associated with abnormal glycosylation (19), which is functionally implicated in the malignant behavior of cancer cells. By staining with fluorescently labeled lectins, we observed a reduction of lectin staining associated with a loss of cell-to-cell adhesion (Fig. 5), suggesting that cholinergic system alterations induced 

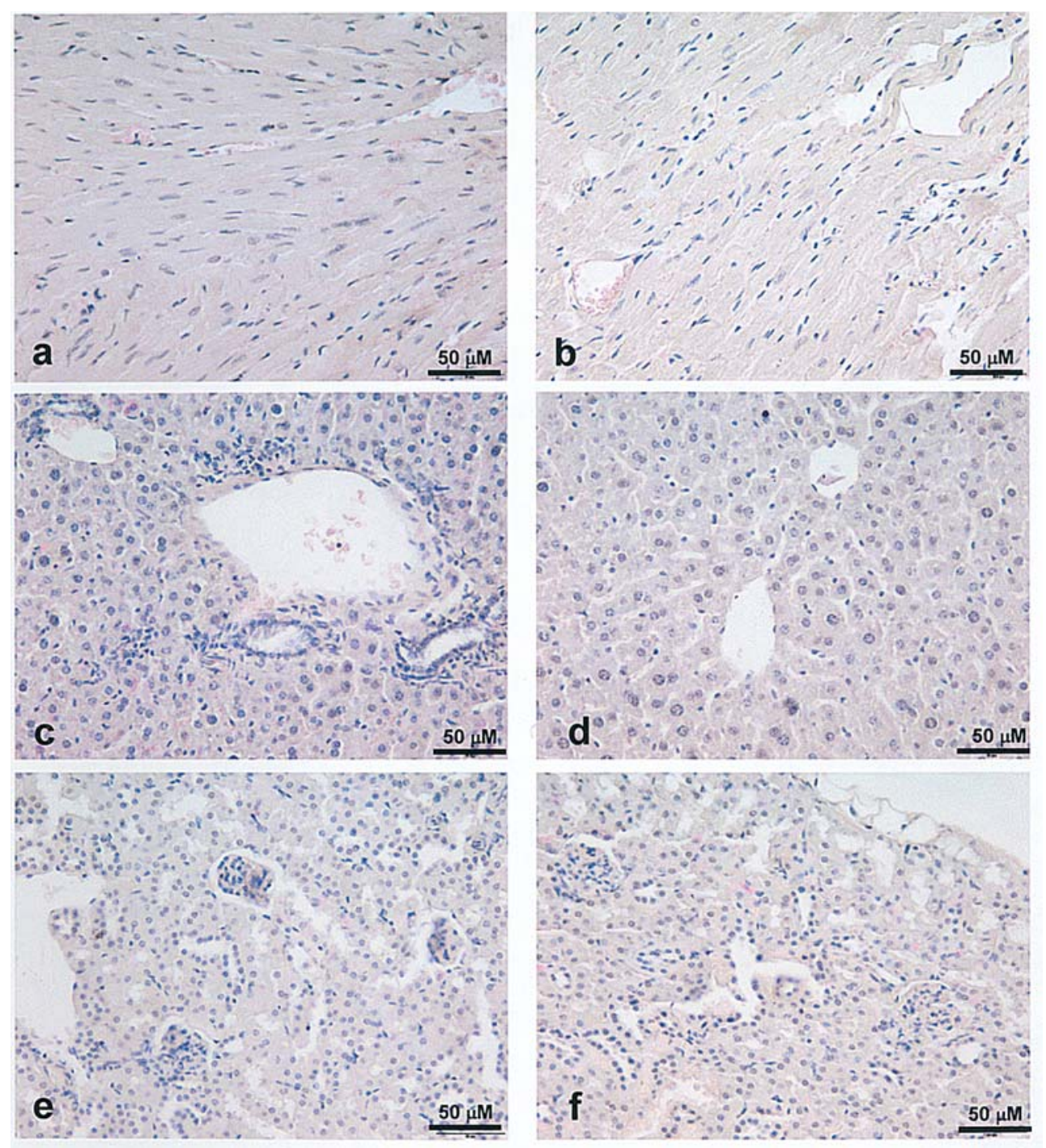

Figure 6. Histological analyses (H\&E) of the major organs for detecting organ toxicity in C57/BL mice: heart (a and b), liver (c and d) and kidney (e and f) respectively treated and untreated with poly-APS salts.

by poly-APS result in loss of cell surface glycoproteins and cell-cell interactions.

In vivo effects of poly-APS. Due to the absence of lethal effects of poly-APS on normal cells in vitro, we assayed them also in vivo to investigate eventual organ toxicity. Intravenous injection at two different doses $(0.5$ and $1 \mathrm{mg} / \mathrm{kg})$ of poly-APS with heparin did not cause any macroscopic toxic effects on the mice. No weight loss in the treated mice was observed, and no toxic effects, such as diarrhea, infection, weakening or obvious behavioral changes, were detected. Subsequent autopsy and histological analyses of selected organs showed that the organ morphology, both at the gross and cytological levels, did not show differences from the controls (Fig. 6).

\section{Discussion}

Here we show that NSCLC cell lines and tissues express a full range of cholinergic molecules indicating that a functional cholinergic signaling system is present in these cells. In cultured A549 cells, the ChAT immuno-reactivity appeared mainly intracytoplasmic, with a distribution gradient from the peri- nuclear to the peripheral region, except for some focal points of cell contact. For these molecules, as according to a recent classification, membrane localization is typical of the neuronal system, while the localization within the cytoplasm is typical for non-neuronal cells (20). Moreover, it is known from the literature that the localization at the cell membrane is one of the conditions for ChAT biosynthetic activity (21), which is regulated by interaction with other proteins related to the cholinergic membrane (22).

Neurotransmission depends on the regulated exocytotic release of chemical transmitter molecules. This requires the packaging of these substances into the specialized secretory vesicles of neurons and neuroendocrine cells, a process mediated by specific vesicular transporters (23). The vesicular ACh transporter (VAChT) is the protein that transports acetylcholine into synaptic vesicles for regulated exocytotic release (24). The presence of this molecule in both NSCLC samples and cell lines demonstrates an active function of $\mathrm{ACh}$ in this tumor type. Moreover, in A549 cells, the immuno-reaction reveals that vesicles are localized in the cytoplasm periphery and under the cell membrane, showing an active exocitosis of the neurotransmitter. The synthesis and secretion of ACh is 
compensated by the presence of the lytic enzyme, AChE, even if ChAT appears to be expressed at higher levels than AChE. In fact, immuno-reactivity and Western blot analysis show higher expression of the synthetic enzyme. Finally, the cells were positive of the membrane receptors of this system, indicating a potential tightly-regulated autocrine loop.

Polymeric alkylpiridinium salts, extracted from the sponge, Reniera sarai, exert a strong inhibition of AChE, the lytic enzyme of the cholinergic system (14). The presence of cholinergic molecules in NSCLC led us to hypothesize that poly-APS could exert possible effects. Poly-APS showed significant NSCLC selective cytotoxicity, where it appeared to induce rapid apoptosis, but was tolerated well by normal cells. To verify the lack of toxicity and the eventual secondary effects of the compound, we injected two different doses of polyAPS intravenously (through the caudal vein) into C57/BL mice. No variations regarding animal weight or macroscopic effects, such as diarrhea, infection, weakening and behavioral alterations, with respect to controls, were observed. Moreover, histological analysis of selected organs that are typical targets of toxic agents showed identical histology with controls.

Malignant transformation is associated with abnormal glycosylation, resulting in the synthesis and expression of altered carbohydrate moieties (19). These recent results have confirmed that these determinants are not merely markers for cancer, but are functionally implicated in the malignant behavior of cancer cells and may be an inevitable consequence of the malignant transformation of cells. We observed that poly-APS interferes with cell-cell adhesion in the suspension culture and alters cell surface glycosylation patterns as assessed by decreased staining with Con-A and WGA lectins.

Our data suggest that the cholinergic system may be an important component of growth regulation in lung cancer, and that interruption of this system through an AChE inhibitor results in selective induction of apoptosis in lung cancer cells. Irinotecan, a camptothecin derivative inhibitor of topoisomerase I that is used in lung cancer treatment and therapy, is also able to inhibit AChE activity $(25,26)$. It is possible that some of the effects of Irinotecan on lung cancer may be due to its ability to inhibit AChE, and selective AChE inhibition may show effectiveness with less toxicity. The apparent lack of toxicity for normal cells in vitro and normal tissues in vivo of the poly-APS AChE inhibitor, coupled with its strong toxicity for NSCLC cells, indicates that this molecule may be a new and innovative chemotherapeutic agent.

\section{Acknowledgements}

This work was supported by grants from the AIRC (Associazione Italiana per la Ricerca sul Cancro), the Ministero della Salute Progetto Finalizzato, the MIUR Progetto Strategico and Progetto FIRB, the Fondi di Ateneo of the University of Insubria, the PNR-Oncologia Citochine \& Chemokine, the Compagnia di San Paolo and the Comitato Interministeriale per la Programmazione Economica (CIPE), SENS-PESTI QLK4-CT-2002-02264 awarded by the European Community (Bruxelles, Belgium) to Carla Falugi, University of Genoa. Drs Falugi and Trombino are fully supported by the grant. The experiments comply with the current European laws of bioethics.

\section{References}

1. Kobzik L: The lung. In: Pathologic Basis of Disease. W.B. Saunders Company, Philadelphia pp697-755, 1999.

2. Weiss W: Epidemiology of lung cancer. In: Comparative Respiratory Tract Carcinogenesis. Schuller HM (ed). CRC Press, Boca Raton, pp1-18, 1983.

3. Cattaneo MG, D'atri F and Vicentini LM: Mechanisms of mitogen-activated protein kinase activation by nicotine in small-cell lung carcinoma cells. Biochem J 328: 499-503, 1997.

4. Sher E, Codignola A, Passafaro M, Tarroni P, Magnelli V, Carbone $\mathrm{E}$ and Clementi F: Nicotinic receptors and calcium channels in small cell lung carcinoma. Functional role, modulation, and autoimmunity. Ann NY Acad Sci 841: 606624,1998

5. Maneckjee R and Minna JD: Opioid and nicotine receptors affect growth regulation of human lung cancer cell lines. Proc Natl Acad Sci USA 87: 3294-3298, 1990.

6. Chini B, Clementi F, Hukovic N and Sher E: Neuronal-type alpha-bungarotoxin receptors and the alpha 5-nicotinic receptor subunit gene are expressed in neuronal and non-neuronal human cell lines. Proc Natl Acad Sci USA 89: 1572-1576, 1992.

7. Song P, Sekhon HS, Jia Y, Keller JA, Blusztajn JK, Mark GP and Spindel ER: Acetylcholine is synthetized by and acts as an autocrine growth factor for small cell lung carcinoma. Cancer Res 63: 214-221, 2003.

8. Pathak AK, Bhutani M, Mohan A, Guleria R, Bal S and Kochupillai V: Non-small cell lung cancer (NSCLC): current status and future prospects. Indian J Chest Dis Allied Sci 46: 191-203, 2004.

9. Fox JL, Rosenzweig KE and Ostroff JS: The effect of smoking status on survival following radiation therapy for non-small cell lung cancer. Lung Cancer 44: 287-293, 2004.

10. Sy SMH, Wong N, Mok TSK, Tsao MS, Lee TW, Tse G, Blackhall FH, Johnson PJ, Anthony P and Yim AY: Genetic alterations of lung adenocarcinoma in relation to smoking and ethnicity. Lung Cancer 41: 91-99, 2003.

11. Tammemagi M, Eskandarian S, Ozcelik H, Tsao MS, Mullen B, Johnston M, McLaughlin J and Casson AG: The association between 553 alterations, ras mutations \& tobacco smoking in non-small cell lung cancer (NSCLC). Lung Cancer 18 (suppl 1): 162-164, 1997

12. Sepcic K, Guella G, Mancini I, Pietra F, Dalla Serra M, Menestrina G, Tubbs K, Macek P and Turk T: Characterization of acetylcholinesterase-active-3-alylpyridinium polymers from the marine sponge Reniera sarai in acqueous solutions. J Natl Prod 60: 991-996, 1997

13. Faimali M, Sepcic K, Turk T and Geraci S: Non-toxic antifouling activity of polymeric 3-alkylpyridinium salts from the Mediterranean sponge Reniera sarai (Pulitzer-Finali). Biofouling 19: 47-56, 2003.

14. Mancini I, Sicurelli A, Guella G, Tur T, Macek P and Sepcic K: Synthesis and bioactivity of linear oligomers related to polymericalkylpyridinium metabolites from the mediterranean sponge reniera sarai. Org Biol Chem 2: 1368-1375, 2004.

15. Sepcic K: Bioactive alkylpyridinium compounds from marine sponges: J Toxicol 19: 139-160, 2000.

16. Sepcic K, et al: Inhibition of acetylcholinesterase by an alkylpyridinium polymer from the marine sponge, Reniera sarai. Biochem Biophys Acta 1387: 217-225, 1998.

17. Sepcic K, Poklar N, Vesnaver G, Fournier D, Turk T and Macek P: Interaction of 3-alkylpyridinium polymers from the sea sponge Reniera sarai with insect acetylcholinesterase. J Protein Chem 18: 251-257, 1999.

18. Raynald P and Pollard HB: Annexins: the problem of assessing the biological role for a gene family of multifunctional calciumand phospholipid-binding proteins. Biochim Biophys Acta 1: 63-93, 1994.

19. Kannagi R, Izawa M, Koike T, Miyazaki K and Kimura N: Carbohydrate-mediated cell adhesion in cancer metastasis and angiogenesis. Cancer Sci 95: 377-384, 2004.

20. Wessler I, Kilbinger H, Bittinger F, Unger R and Kirkpatrick CJ: The non-neuronal cholinergic system in humans: expression, function and pathophysiology. Life Sci 72: 2055-2061, 2003.

21. Dobransky T and Rylett RJ: Functional regulation of choline acetyltransferase by phosphorylation. Neurochem Res 28: 537-542, 2003. 
22. Gabrielle P, Jeana M and Lorenza EC: Cytosolic choline acetyltransferase binds specifically to cholinergic plasma membrane of rat brain synaptosomes to generate membrane-bound enzyme. Neurochem Res 28: 543-549, 2003.

23. Varoqui H and Erickson JD: Vesicular neurotransmitter transporters. Potential sites for the regulation of synaptic function. Mol Neurobiol 15: 165-191, 1997.

24. Roghani A, Feldman J, Kohan SA, Shirzadi A, Gunderson CB, Brecha $\mathrm{N}$ and Edwards RH: Molecular cloning of a putative vesicular transporter for acetylcholine. Proc Natl Acad Sci USA 91: 10620-10624, 1994.
25. Doods HM, Hanrhan J and Rivory LR: The inhibition of acetylcholinesterase by irinotecan and related camptothecins: key structural properties and experimental variables. Anticancer Drugs Des 16: 239-246, 2001.

26. Fukuda M, Oka M, Soda H, et al: Phase II study of irinotecan combined with carboplatin in previously untreated non-smallcell lung cancer. Cancer Chemother Pharmacol 54: 573-577, 2004. 